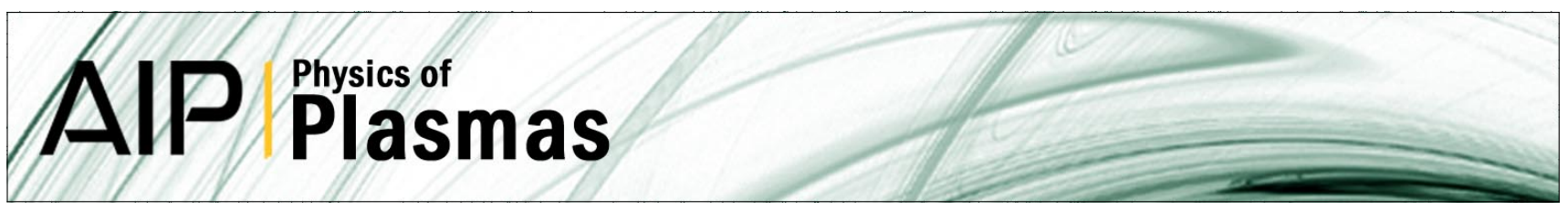

\title{
Short wavelength ion temperature gradient turbulence
}

J. Chowdhury, S. Brunner, R. Ganesh, X. Lapillonne, L. Villard et al.

Citation: Phys. Plasmas 19, 102508 (2012); doi: 10.1063/1.4759458

View online: http://dx.doi.org/10.1063/1.4759458

View Table of Contents: http://pop.aip.org/resource/1/PHPAEN/v19/i10

Published by the American Institute of Physics.

\section{Related Articles}

Analysis of the influence of external biasing on Texas Helimak turbulence

Phys. Plasmas 20, 022310 (2013)

Transport of radial heat flux and second sound in fusion plasmas

Phys. Plasmas 20, 022307 (2013)

On numerical turbulence generation for test-particle simulations

Phys. Plasmas 20, 022302 (2013)

The rapid acceleration model and the growth rate of a turbulent mixing zone induced by Rayleigh-Taylor instability

Phys. Fluids 25, 015118 (2013)

Coherent structures, intermittent turbulence, and dissipation in high-temperature plasmas

Phys. Plasmas 20, 012303 (2013)

\section{Additional information on Phys. Plasmas}

Journal Homepage: http://pop.aip.org/

Journal Information: http://pop.aip.org/about/about_the_journal

Top downloads: http://pop.aip.org/features/most_downloaded

Information for Authors: http://pop.aip.org/authors

\section{ADVERTISEMENT}

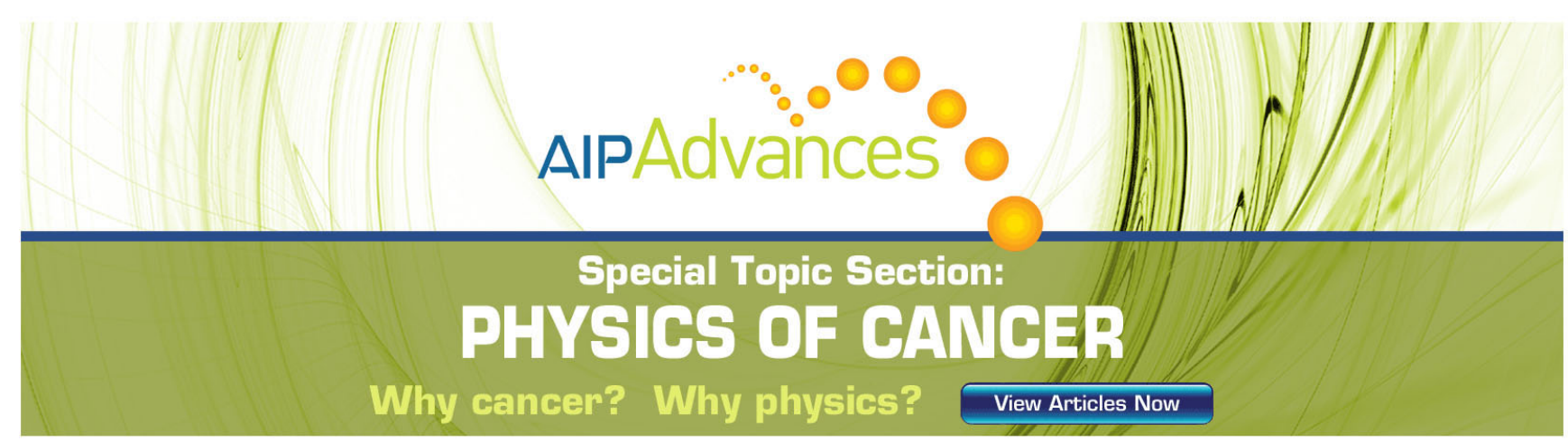




\title{
Short wavelength ion temperature gradient turbulence
}

\author{
J. Chowdhury, ${ }^{1}$ S. Brunner, ${ }^{2}$ R. Ganesh, ${ }^{1}$ X. Lapillonne, ${ }^{2}$ L. Villard,${ }^{2}$ and F. Jenko ${ }^{3}$ \\ ${ }^{1}$ Institute for Plasma Research, Bhat, Gandhinagar, India \\ ${ }^{2}$ CRPP, Association EURATOM-Confédération Suisse, EPFL, 1015 Lausanne, Switzerland \\ ${ }^{3}$ Max-Planck-Institut für Plasmaphysik Boltzmannstr. 2, D-85748 Garching, Germany
}

(Received 28 May 2012; accepted 3 October 2012; published online 17 October 2012)

The ion temperature gradient (ITG) mode in the high wavenumber regime $\left(k_{y} \rho_{s}>1\right)$, referred to as short wavelength ion temperature gradient mode (SWITG) is studied using the nonlinear gyrokinetic electromagnetic code GENE. It is shown that, although the SWITG mode may be linearly more unstable than the standard long wavelength $\left(k_{y} \rho_{s}<1\right)$ ITG mode, nonlinearly its contribution to the total thermal ion heat transport is found to be low. We interpret this as resulting from an increased zonal flow shearing effect on the SWITG mode suppression. (C) 2012 American Institute of Physics. [http://dx.doi.org/10.1063/1.4759458]

\section{INTRODUCTION}

Mitigation of anomalous transport of particles and energy has been a longstanding issue in the field of magnetically confined fusion plasmas. The loss of particles and heat from the core of the toroidal device, holding ions and electrons in plasma state, reduces the efficiency of such a system and thus remains to be one of the hindrances on the way to the construction of fusion reactors. Various low frequency and fine scale length unstable drift waves ${ }^{1}$ are considered to be responsible for degradation of confinement, leading to the so-called anomalous loss of particles and energy. The free energy available in the density and temperature inhomogeneities inherent to a confined plasma is known to feed these instabilities and leads to ion and electron losses through different channels characterized by different length scales. The heat loss via the electron channel takes place mainly through the electron temperature gradient $(\mathrm{ETG})^{2-4}$ mode, toroidal universal drift mode, ${ }^{5,6}$ and trapped electron mode (TEM). ${ }^{7-9}$ The heat loss via the ion channel, on the other hand, is observed to take place by the ion temperature gradient (ITG) ${ }^{1,10,11}$ mode.

However, of late, a new mode propagating in the ion diamagnetic direction, on the intermediate scale between the ITG and ETG modes with $k_{\perp} \rho_{s}>1$, where $k_{\perp}$ and $\rho_{s}$ are the perpendicular wave number and ion Larmor radius evaluated at the sound speed, respectively, has been identified ${ }^{12-16}$ linearly. This mode is found to be driven by the temperature gradient of ions in the presence of the Landau resonance/ inverse resonance in a slab geometry and by the toroidal drift resonance in a toroidal geometry, in combination with the non-monotonic behavior of the mode frequency with respect to the perpendicular wave number. Because of its occurrence in the short wavelength limit and due to the non-adiabaticity of ions, the mode is referred to as the short wavelength ion temperature gradient (SWITG) mode to distinguish it from the standard ITG mode at longer wavelength. It is generally speculated that in the limit $\left(k_{\perp} \rho_{s}\right)^{2} \gg 1$ there should be no mode intrinsic to the ion non-adiabaticity, since ion dynamics in this limit is expected to be adiabatic-like. However, if the scale length of inhomogeneity is such that $\omega_{* i}$, the ion diamagnetic drift frequency, becomes larger than the mode frequency $\omega$, there can be an instability related to the pressure inhomogeneity of the ions even in this shorter wavelength limit. ${ }^{12-16}$

Initially, the mode was thought to be of hybrid type, ${ }^{13,14}$ requiring both $\eta_{i}$ and $\eta_{e}$ (ratio of the density to temperature scale length of the ions and electrons, respectively) to be above a threshold. Later a parametric study by Gao et al. ${ }^{15}$ demonstrated that the electron non-adiabaticity is not an essential ingredient for the mode to develop. Effects of magnetic shear on this mode have been studied in the sheared slab geometry and found to have strong stabilizing impact on the mode. ${ }^{18}$ Dependence of the critical gradient on the various physical parameters such as the electron to ion temperature ratio, toroidicity, magnetic shear, and safety factor has been studied for this mode linearly. ${ }^{19}$ It is to be noted that the typical double hump behavior of growth rate (first hump corresponding to the standard ITG mode and second hump corresponding to the SWITG mode) was pointed out a long way back by Pu et al. ${ }^{20}$ while studying the ion mixing mode. Thus, it is inherently an ion mode and exists even if the electrons are adiabatic; ${ }^{15}$ toroidicity has a strong stabilizing effect on the mode; magnetic shear has a destabilizing effect and the growth rate is approximately proportional to $\sqrt{|\hat{s}|},{ }^{13}$ where $\hat{s}$ stands for magnetic shear; similar to the standard ITG, it is also stabilized by a modest $\alpha$, the ballooning parameter; non-adiabatic circulating electron dynamics provide destabilization; $E \times B$ flow shear has a strong stabilizing effect on the mode. ${ }^{18}$ With adiabatic electrons, in the limit $\omega_{n i}>\omega>\left(\omega_{d i}+k_{\|} v_{\|}\right)$, where $\omega_{d i}$ is the ion magnetic drift frequency, the quasineutrality equation, in the frame of a local gyrokinetic formulation, gives the following dispersion relation for SWITG: ${ }^{15}$

$$
\omega=\left(\frac{\tau}{\tau+1}\right)\left(\frac{\eta_{i}}{2}-1\right) \omega_{n i} I_{0}\left(k_{\perp}^{2} \rho_{s}^{2}\right) \exp \left(-k_{\perp}^{2} \rho_{s}^{2}\right),
$$

where $\omega_{n i}=-\left(v_{t h i} / L_{n}\right)\left(k_{\perp} \rho_{s}\right)$ is the ion diamagnetic drift frequency, $\tau=T_{e} / T_{i}, L_{n}$ is the density scale length, and $I_{0}$ is the modified Bessel function of order zero.

Although detailed studies have been pursued in the linear behaviour (for example, one can see Refs. 13, 15, 16, and 18), 
there is to our knowledge no systematic nonlinear study on the SWITG mode. It is therefore important to investigate how this mode behaves nonlinearly and if there is any significant contribution of this mode to the net ion transport in the core of the system. To this end, we carry out a systematic nonlinear study of the mode using the flux tube version of the well benchmarked, massively parallel, nonlinear, gyrokinetic code GENE. ${ }^{8,21,22}$ We have found that although linearly the growth rate of the SWITG mode is equally strong as the one of the standard ITG mode and the $k_{y}$ spectrum of the nonlinear turbulent state bears signature of the mode, there is only a very weak contribution to the overall ion thermal transport from the SWITG mode.

The remainder of the paper is arranged as follows. In Sec. II, the simulation model is presented. Section III discusses the results. Finally, conclusions are drawn in Sec. IV.

\section{THE MODEL}

\section{A. Coordinate system}

The set of equations for the electrostatic case are cast in the Clebsch-type field-aligned coordinate system $(x, y, z)$, such that $(x, y)$ represents the plane perpendicular to the magnetic field given by $\mathbf{B}=B_{0} \nabla x \times \nabla y$, where $x$ stands for the flux surface label, simply the radial coordinate in units of length, $y$ is the binormal direction in units of length that labels the field line on a given flux surface and $z$ is the straight field line poloidal angle that labels the position along a field line and thus represents the parallel direction. $B_{0}$ is the reference magnetic field on axis. The velocity space is represented by $\left(v_{\|}, \mu\right)$, where, $v_{\|}$is the velocity component parallel to $\vec{B}$ and $\mu=m v_{\perp}^{2} / 2 B$ stands for the magnetic moment.

As the present nonlinear simulation uses the local version of GENE, the system considered is therefore a flux tube, where system size is a box of dimension $L_{x} \times L_{y} \times L_{z}$ in configuration space as well as $L_{v_{\|}} \times L_{\mu}$ in velocity space. In the flux-tube model, all equilibrium profiles and their gradients are approximated to be constants. In the perpendicular direction, i.e., radial $x$ and binormal $y$ directions, periodic boundary conditions are applied.

\section{B. Discretization scheme}

The discretization scheme used is based on the so-called "method of lines," that is, the phase space operators are discretized first, and then the resulting ordinary differential equations for the phase space grid values of the particle distribution(s) are solved as an initial value problem using a fourth order Runge-Kutta scheme. The differential operators related to the field-line following coordinate $z$ or the parallel velocity $v_{\|}$are discretized using a fourth-order finite difference scheme. The invariance of profiles and their gradients, as well as the periodic boundary conditions in the perpendicular $x$ and $y$ directions allow one to treat $x$ and $y$ in the framework of a pseudo-spectral approach, that is, all linear terms as well as spatial derivatives are evaluated in $k_{x}-k_{y}$ space, while the nonlinearities are computed in real space with the help of Fourier transforms and a proper dealiasing method. The velocity space integrations are performed using Gauss and trapezoidal rules in $\mu$ and $v_{\|}$space, respectively. For the time stepping, the fourth-order explicit Runge-Kutta method is used.

\section{C. $\delta$ f splitting}

In order to separate the macroscopic evolution of the plasma from the microturbulence, the full ion distribution function is considered to be composed of two parts, namely, the static background distribution function $f_{0}$ and a perturbed part $f_{1}$ of the order of $\left|f_{1} / f_{0}\right| \sim \rho_{\text {ref }} / L_{\text {ref }} \ll 1$, where $\rho_{\text {ref }}$ and $L_{r e f}$, respectively, stand for a reference Larmor radius and equilibrium scale length. We consider

$$
f_{0 i}\left(v_{\|}, \mu\right)=\frac{n_{0}}{\left(2 \pi T_{i} / m_{i}\right)^{3 / 2}} \exp \left[-\frac{m_{i} v_{\|}^{2} / 2+\mu B}{T_{i}}\right],
$$

to be a local Maxwellian distribution function, $n_{0}$ and $T_{i}$ being the background density and ion temperature. The perturbed distribution function $f_{1}$ is, on the other hand, a function of the five gyrocenter phase space coordinates $\left(x, y, z, v_{\|}, \mu\right)$ and time $t$.

\section{Normalizations}

The normalizations of the various equilibrium and fluctuating quantities are displayed in Table I, where $v_{T i}=$ $\sqrt{2 T_{i} / m_{i}}$ and $c_{s}=\sqrt{Z_{i} T_{e} / m_{i}}$ stand, respectively, for the ion thermal velocity and sound speed, $\rho_{s}=c_{s} / \Omega_{i}$ is the ion Larmor radius at the sound speed and $\Phi_{1}$ is the electrostatic potential related to the fluctuations.

The spirit behind the choice of the normalizations is to make each normalized quantity of order unity. Furthermore, all gradient scale lengths of the equilibrium quantities, viz., $n_{0}, T_{i}, B$, are normalized to $R_{0}$.

\section{E. Model equations}

With the above defined coordinate system and normalizations, and with the suffix defining particle type and the hat for normalized quantities being dropped to ease the notation, the gyrokinetic equation for ions can formally be written as

$$
\begin{aligned}
\frac{\partial f_{1}}{\partial t}+ & {\left[\frac{1}{L_{n}}+\frac{1}{L_{T}}\left(v_{\|}^{2}+\mu B-3 / 2\right)\right] f_{0} \frac{\partial \bar{\Phi}_{1}}{\partial y} } \\
+ & {\left[\frac{\partial \bar{\Phi}_{1}}{\partial x} \frac{\partial f_{1}}{\partial y}-\frac{\partial \bar{\Phi}_{1}}{\partial y} \frac{\partial f_{1}}{\partial x}\right]+\frac{1}{B} \frac{\mu B+2 v_{\|}^{2}}{\sigma}\left(K_{x} G_{x}+K_{y} G_{y}\right) } \\
& +\frac{v_{\|}^{2} \beta}{\sigma B} \frac{d p}{d x} G_{y}+\alpha \frac{v_{\|}}{J B} G_{z}-\frac{\mu \alpha}{2 J B} \frac{\partial f_{1}}{\partial v_{\|}} \frac{\partial B}{\partial z}=0,
\end{aligned}
$$

where $G_{j}=\partial_{j} f_{1}-\left(\sigma / v_{\|}\right) \partial_{j} \bar{\Phi}_{1} \partial f_{0} / \partial v_{\|}$for $j=x, y, z, \alpha_{i}=v_{T i} / c_{s}$ and $\sigma_{i}=Z_{i} T_{e} / T_{i}$ and $\bar{\Phi}_{1}=J_{0}(\lambda) \Phi_{1}$ is the gyroaveraged potential with $\lambda^{2}=k_{\perp}^{2}(2 \mu / B)$ and $J_{0}$ being the Bessel

TABLE I. Normalization of dependent variables

\begin{tabular}{ccc}
\hline \hline$\hat{f}_{i 0}$ & $\hat{f}_{i 1}$ & $\hat{\Phi}_{1}$ \\
$\frac{f_{i 0} v_{T i}^{3}}{n_{0}}$ & $\left(\frac{f_{i 1} v_{T i}^{3}}{n_{0}}\right)\left(\frac{R_{0}}{\rho_{s}}\right)$ & $\frac{e \Phi_{1}}{T_{e}}\left(\frac{R_{0}}{\rho_{s}}\right)$ \\
\hline
\end{tabular}


function of order zero. Also, $1 / L_{T}=-d(\ln \mathrm{T}) / d x$ and $1 / L_{n}$ $=-d(\ln \mathrm{n}) / d x$ are the inverse gradient scale lengths of temperature and density, respectively. The second term in Eq. (2) thus represents the equilibrium gradients that drive the instability. The third term is the nonlinear $E \times B$ drift term. The fourth and fifth terms contain the effect of magnetic curvature and $\nabla B$, with

$$
K_{x}=-\frac{g^{x x} g^{y z}-g^{y x} g^{x z}}{B^{2}} \frac{\partial B}{\partial z},
$$

and

$$
K_{y}=\frac{\partial B}{\partial x}-\frac{g^{x y} g^{y z}-g^{y y} g^{x z}}{B^{2}} \frac{\partial B}{\partial z},
$$

where the metric tensor elements are defined as $g^{i j}=\nabla u^{i}$. $\nabla u^{j}, u^{i}, u^{j}=x, y, z$. The total plasma pressure appearing with a radial derivative in the fifth term is normalized to $p_{\text {ref }}=n_{0} T_{e}$, the reference pressure (at the center of the flux tube). Also $\beta=2 p_{\text {ref }} \mu_{0} / B^{2}$, where $\mu_{0}$ is the permeability in vacuum. Finally, the last two terms take into account the effect of particle trapping in the low magnetic field side of the tokamak and include the Jacobian $J=J^{x y z}=[(\nabla x$ $\times \nabla y) \cdot \nabla z]^{-1}$. Integrating the perturbed distribution $f_{1}$ over velocity, solution to Eq. (2), provides the perturbed ion density, which can be inserted in the quasineutrality condition with adiabatic electrons, thus leading to the following equation:

$$
Z^{2} \tau\left[1-\Gamma_{0}(b)\right] \Phi_{1}=\pi Z B \int J_{0}(\lambda) f_{1} d v_{\|} d \mu-\left(\Phi_{1}-\left\langle\Phi_{1}\right\rangle\right) .
$$

Equation (3) can then be solved numerically, to get the self consistent electrostatic potential $\Phi_{1}$. Note that in Eq. (3), one defines $\tau=T_{e} / T_{i}, \quad b=\left[1 /\left(Z_{i}^{2} \tau B^{2}\right)\right] k_{\perp}^{2}, \quad k_{\perp}^{2}=g^{x x} k_{x}^{2}+g^{y y} k_{y}^{2}$ $+g^{x y} k_{x} k_{y}$ and $\Gamma_{0}(b)=\exp (-b) I_{0}(b)$ is the scaled modified Bessel function. The term $\left\langle\Phi_{1}\right\rangle$ represents the flux surface averaged value of $\Phi_{1}$. Note that in a flux-tube simulation, the $x$-dependence of all equilibrium quantities across the simulation domain is neglected. Thus, all coefficients relative to the magnetic equilibrium $\left[B(z), J(z), K_{x}(z), K_{y}(z), g^{i j}(z)\right]$ as well as equilibrium profiles and their gradients $\left(1 / L_{n}, 1 / L_{T}\right)$ are independent of $x$ and represent values at the flux tube center defined by $x=x_{0}$.

\section{F. Geometry}

The GENE code is capable to interface with the general geometry MHD equilibrium obtained from the numerical solution of the Grad-Shafranov equation. It can, in addition, also be run using an ad hoc analytical model which is an approximate solution of the Grad-Shafranov equation in the limit of axisymmetric, circular, and concentric flux surfaces. In the present investigation, we use this ad hoc equilibrium, which is defined in the $(r, \theta, \phi)$ (radial, poloidal, and toroidal) coordinate system, related to the cylindrical coordinate system $(R, z, \phi)$ by the relations $R=R_{0}+r \cos \theta=R_{0}(1$ $+\epsilon \cos \theta)$ and $Z=r \sin \theta$, with $R_{0}$ the major radius of the toroidal coordinate system and $\epsilon=r / R_{0}$ being the inverse aspect ratio. The axisymmetric magnetic field can always be written as $\mathrm{B}=\nabla \phi \times \nabla \psi+R B_{\phi} \nabla \phi$, where in the ad hoc model $B_{\phi}=R_{0} B_{0} / R, \Psi=\Psi(r)$ and $d \Psi / d r=r B_{0} / \bar{q}$. The function $\bar{q}(r)$ is related to the true safety factor $q$ by the relation

$$
q(r)=\frac{1}{2 \pi} \int_{0}^{2 \pi} \frac{\mathbf{B} \cdot \nabla \phi}{\mathbf{B} \cdot \nabla \theta} d \theta=\frac{\bar{q}(r)}{\sqrt{1-\epsilon^{2}}} .
$$

More details related to this ad hoc model are found in Ref. 24.

\section{G. Physical parameters}

Our main focus is to study the nonlinear short wavelength ion temperature gradient mode using the flux tube version of GENE. Note that a detailed parametric study of the linear SWITG mode has been carried out in Ref. 16 using the global spectral gyrokinetic code EM-GLOGYSTO ${ }^{17}$ considering $\rho^{*}=\rho_{s} / a=0.017$, inverse aspect ratio $a / R_{0}$ $=0.25$, and steepest gradients at $s=r / a=0.6$. Inspired by this linear study, we have chosen the inverse aspect ratio $r_{0} / R_{0}=0.15$ in the present nonlinear flux tube simulations, corresponding to the limit $\rho^{*} \rightarrow 0$. Also in the present simulation, we have considered $\tau=T_{e} / T_{i}=1, q_{0}=q\left(r_{0}\right)=2.0$, $\hat{s}=1.0$ and $\eta_{i}=L_{n} / L_{T_{i}}=2.5$, where $L_{n}$ and $L_{T i}$ are, respectively, the density and temperature gradient scale lengths. The density gradient is varied over $R / L_{n}=5-10$. Note that these kind of steep profiles are usually found in transport barriers. ${ }^{25}$

\section{H. Numerical parameters}

The simulation is carried out in a box of size $L_{x} \times L_{y}$ $=133.3 \rho_{s} \times 209.4 \rho_{s}$ in the perpendicular direction. One connection length of $2 \pi R q$ is considered in the parallel direction $\left(L_{z}=2 \pi\right)$. This 3 -dimensional spatial domain is resolved by using $200 \times 120 \times 16$ grid points, respectively, in the $x, y$, and $z$ directions. The velocity space, on the other hand, is resolved with $32 \times 16$ grid points, respectively, for $v_{\|}$and $\mu$ with the limit for $v_{\|}$from -3 to +3 , and 0 to 9 for $\mu$ in normalized units (see Table II).

\section{RESULTS}

In this section, we will delineate our results for linear and nonlinear runs obtained using the flux tube version of GENE and the physical and numerical parameters introduced above.

\section{A. Linear spectrum}

We have first carried out a linear simulation with parameters considered above. Figure 1(a) shows the real frequency

TABLE II. Normalization of independent variables.

\begin{tabular}{lccccc}
\hline \hline$\hat{t}$ & $\hat{x}$ & $\hat{y}$ & $\hat{z}$ & $\hat{v}_{\|}$ & $\hat{\mu}$ \\
$c_{s} t / R_{0}$ & $x / \rho_{s}$ & $y / \rho_{s}$ & $z$ & $v_{\|} / v_{t h i}$ & $\mu B_{0} / T_{i}$ \\
\hline \hline
\end{tabular}




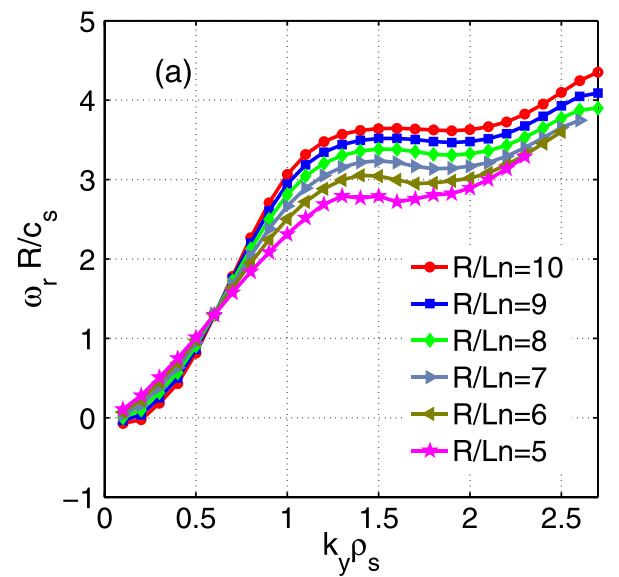

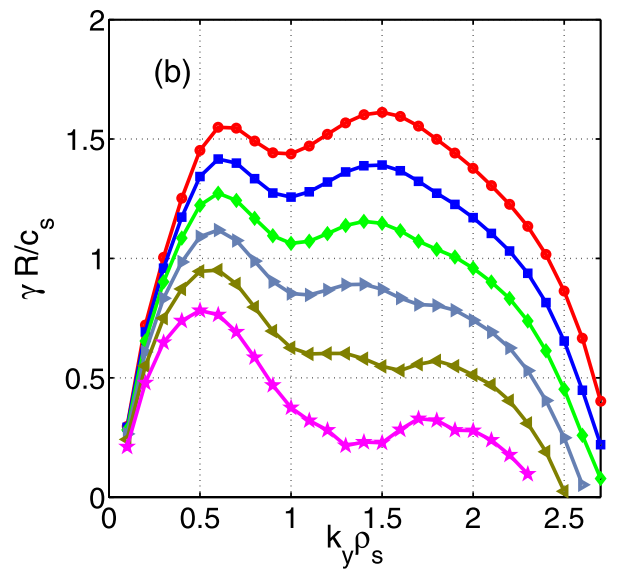

FIG. 1. (a) Real frequency $\omega_{r}$ and (b) growth rate $\gamma$ versus wave number $k_{y} \rho_{s}$ of the linear SWITG for different values of $R / L_{n}$. Here $\eta_{i}=2.5, q=2.0, \hat{s}=1.0$, $\tau=1.0$ in these simulations. versus $k_{y} \rho_{s}$ for different values of $R / L_{n}$ ranging from 5 to 10. The real frequency rises monotonically with $k_{y} \rho_{s}$ for $k_{y} \rho_{s} \leq 1$ but then remains virtually constant for $1 \leq k_{y} \rho_{s}$ $\leq 2$. It is clear from the expression of the dispersion relation, Eq. (1), that the mode frequency $\omega$ behaves as $\omega_{n i} I_{0}\left(k_{\perp}^{2} \rho_{s}^{2}\right)$ $\exp \left(-k_{\perp}^{2} \rho_{s}^{2}\right)$ which for small $k_{\perp}^{2} \rho_{s}^{2}$ scales as $k_{\perp} \rho_{s}$ and for larger $k_{\perp}^{2} \rho_{s}^{2}$ scales almost as a constant as $I_{0}\left(k_{\perp}^{2} \rho_{s}^{2}\right) \exp$ $\left(-k_{\perp}^{2} \rho_{s}^{2}\right) \rightarrow 1 / \sqrt{2 \pi\left(k_{\perp}^{2} \rho_{s}^{2}\right)}=1 /\left(\sqrt{2 \pi} k_{\perp} \rho_{s}\right)$ for $k_{\perp} \rho_{s} \gg 1$ (recall that $\omega_{n i} \sim k_{\perp} \rho_{s}$ ). Figure 1(b) displays the growth rate for the same scan. It exhibits two peaks for all considered values of $R / L_{n}$ in contrast to the single peak around $k_{y} \rho_{s}$ $\simeq 0.6$ generally observed in the linear analysis of the standard ITG modes. The second peak appears around $k_{y} \rho_{s} \simeq 1.5$ and is characteristic of the SWITG mode. Regarding the growth rate in toroidal geometry, it is the toroidal magnetic drift term $\omega_{d i}$ of the ions, resonating with the mode frequency $\omega$ which gives rise to the double hump behavior. It is to be noted that $\omega_{d i} \sim\left(L_{n} / R\right) \omega_{n i}$ and thus scales as $k_{\perp} \rho_{s}$. Therefore, both $\omega$ and $\omega_{d i}$ at first increase for small $k_{\perp} \rho_{s}$ and then $\omega$ saturates while $\omega_{d i}$ still grows as $k_{\perp} \rho_{s}$ increases. It is important to note that the SWITG mode is also subject to finite Larmor radius effects. The finite Larmor radius stabilization (FLR) of the SWITG mode can be inferred from the nonadiabatic part of the ion density response. At very high $k_{\perp} \rho_{s}$, $\left|\omega_{d i}\right| \gg|\omega|$ and the nonadiabatic part of the perturbed ion density can be reduced to ${ }^{13,15}$

$$
\tilde{n}_{i}^{n a}=\frac{q_{i}}{T_{i}} \tilde{\phi} \frac{\omega_{n i}\left(\eta_{i} / 2-1\right)}{\omega_{d i}} I_{0}\left(k_{\perp}^{2} \rho_{s}^{2}\right) \exp \left(-k_{\perp}^{2} \rho_{s}^{2}\right),
$$

which for large $k_{\perp} \rho_{s}$ will decrease according to

$$
\begin{aligned}
\frac{\omega_{n i}}{\omega_{d i}} I_{0}\left(k_{\perp}^{2} \rho_{s}^{2}\right) \exp \left(-k_{\perp}^{2} \rho_{s}^{2}\right) & \sim \frac{R}{L_{n}} I_{0}\left(k_{\perp}^{2} \rho_{s}^{2}\right) \exp \left(-k_{\perp}^{2} \rho_{s}^{2}\right) \\
& \sim \frac{R}{L_{n}} \frac{1}{\sqrt{2 \pi} k_{\perp} \rho_{s}},
\end{aligned}
$$

as $k_{\perp}^{2} \rho_{s}^{2}$ increases. It is apparent that the second hump is equally strong as the first hump for higher value of $R / L_{n}$ or even slightly more dominant for $R / L_{n} \geq 10$. It also is present for lower values of $R / L_{n}$ albeit less pronounced and finally vanishes at values of $R / L_{n}$ typical of the standard ITG mode $\left(R / L_{n}<5\right)$.

\section{B. Nonlinear spectra}

Till date, there have been very few nonlinear studies of the SWITG mode. The study of the SWITG mode turbulence nonlinearly and its contribution to the net thermal ion heat transport may therefore add valuable physics insight and enhance our understanding of the ITG driven turbulence in general. Here, we try to address this issue. We have adopted two cases in the present nonlinear study. Case (I): $R / L_{n}=10$, where the peak in the linear growth rate corresponding to the SWITG (at $k_{y} \rho_{s} \sim 1.5$ ) mode is as pronounced as that at lower $k_{y}\left(k_{y} \rho_{s} \sim 0.6\right)$ corresponding to the standard ITG mode. Case (II): $R / L_{n}=5$, where the linear growth rate of SWITG at $k_{y} \rho_{s}>1$ is much weaker than that of the standard ITG mode at $k_{y} \rho_{s}<1$. The time evolution of the nonlinear ion heat flux given by the relation $Q=\left\langle\int(1 / 2) m v^{2} \vec{v}_{E}\right.$ - $\left.\nabla x f_{1} d^{3} v\right\rangle$, normalized with respect to $Q_{\text {norm }}=p_{\text {ref }} c_{s} \rho_{s}^{2} / R^{2}$, where $p_{\text {ref }}$ is the equilibrium pressure, is shown in Fig. 2 for the parameters described in Secs. II G and II H and for the two cases of the parameter $R / L_{n}$. Note that $\langle A\rangle=\int J^{x y z}(z) A(z) d z /$ $\int J^{x y z}(z) d z$ stands for the flux-averaged quantity $A$, where $J^{x y z}$ is the Jacobian relative to the coordinate system $(x, y, z)$. Also, $v$ and $\vec{v}_{E} \cdot \nabla x$ are, respectively, the particle velocity and the radial component of the gyroaveraged $E \times B$ drift velocity, and $f_{1}$ is the perturbed distribution function. It is clear that the heat

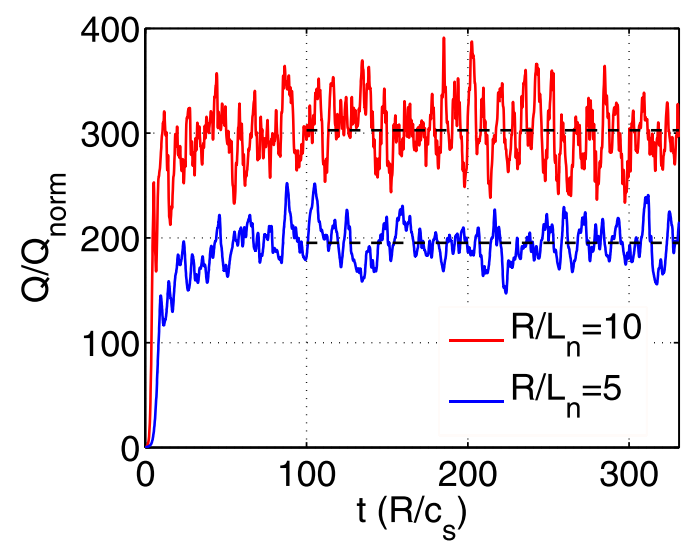

FIG. 2. Time (in units of $R / c_{s}$ ) evolution of ion heat flux $Q$ normalized by $Q_{\text {norm }}=p_{\text {ref }} c_{s} \rho_{s}^{2} / R^{2}$ for SWITG simulation, with $R / L_{n}=5$ and $R / L_{n}=10$. The black dashed lines represent the time average of the heat flux between $t=100 R / c_{s}$ and $t=330 R / c_{s}$. For $R / L_{n}=5$ the average $Q=195 Q_{\text {norm }}$ and for $R / L_{n}=10$ the average $Q=302 Q_{\text {norm }}$. 
flux in the case of $R / L_{n}=10$ is higher than that in the case of $R / L_{n}=5$. This is consistent with the linear results where the modes for $R / L_{n}=10$ exhibit higher growth rates than for $R / L_{n}=5$ at both scales, i.e., $k_{y} \rho_{s}<1$ and $k_{y} \rho_{s}>1$, corresponding, respectively, to the standard ITG and SWITG modes. The ratio of growth rates for $R / L_{n}=5$ compared to $R / L_{n}=10$ is even weaker for the SWITG scales than for the ITG scales. The time averaged heat flux between $t=100 R / c_{s}$ and $t=330 R / c_{s}$ is estimated at $302 Q_{\text {norm }}$ and $195 Q_{\text {norm }}$, respectively, for the nonlinear simulations with $R / L_{n}=10$ and $R / L_{n}=5$. Figure 3(a) depicts the time averaged (from $t=20$ to $\left.t=330 R / c_{s}\right)$ spectrum $Q\left(k_{y}\right)$ of the heat flux for the $R / L_{n}=10$ case, normalized with respect to $Q_{\text {norm }}$, over the entire $k_{y} \rho_{s}$ spectrum considered in the simulation. For comparison, we also plot in the same figure the time averaged (from $t=20$ to $t=330 R / c_{s}$ ) spectrum of the heat flux $Q\left(k_{y}\right)$ measured from the simulation with $R / L_{n}=5$. The corresponding log-log plots for both simulations are shown in Fig. 3(b). It is quite clear that the $Q\left(k_{y}\right)$ spectrum exhibits a strong peak around $k_{y} \rho_{s} \simeq 0.1$ for both values of $R / L_{n}$. However, the peak corresponding to $R / L_{n}=5$ is significantly lower than the one corresponding to $R / L_{n}=10$, in conformity with the observed heat flux displayed in Fig. 2. These plots also illustrate that on the higher side of the $k_{y}$ spectrum corresponding to the SWITG mode, the contribution to the net ion heat flux is very low compared to the contribution from the standard ITG mode on the lower side of the $k_{y}$ spectrum. The characteristic of the SWITG mode,
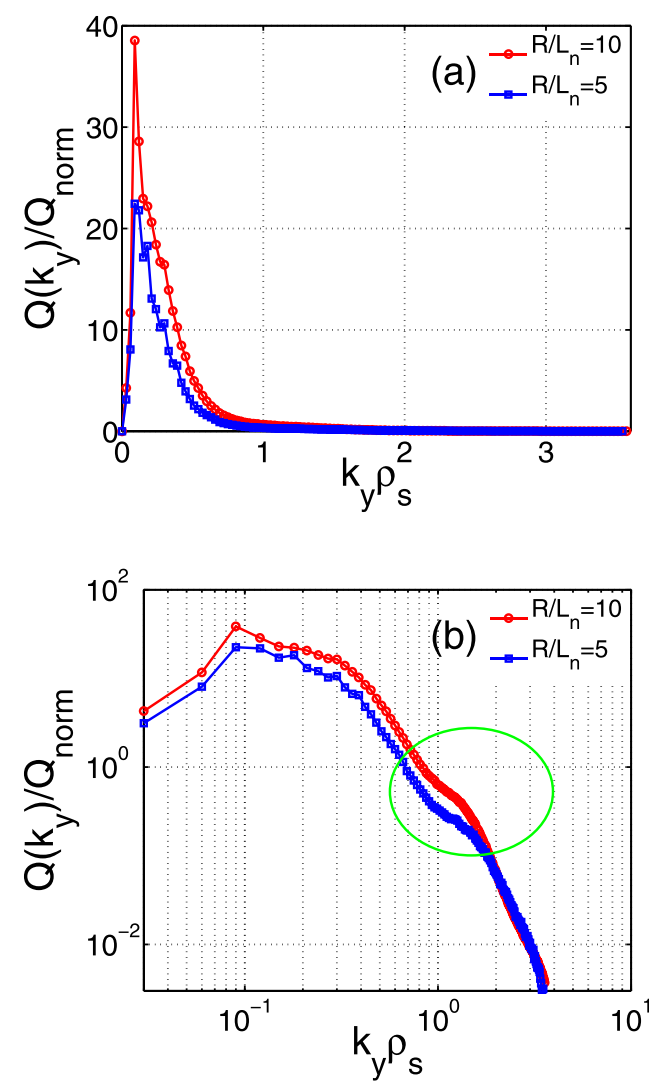

FIG. 3. Time averaged ion heat flux versus normalized wavenumber $k_{y}$ for $R / L_{n}=5$ and $R / L_{n}=10$ in (a) lin-lin plot and (b) log-log plot. The circle shows the region where SWITG mode is unstable. $Q$ is normalized with respect to $Q_{\text {norm }}=p_{\text {ref }} c_{s} \rho_{s}^{2} / R^{2}$. though weak, is nevertheless still visible from Fig. 3(b). The hump corresponding to the SWITG mode (encircled in Fig. 3 (b)) centers around $k_{y} \rho_{s}=1.5$, reflecting that there is another source of instability in this region of the spectrum. Since the area under a usual log-log curve has no direct physical meaning, one may plot $k_{y} Q\left(k_{y}\right)$ vs $k_{y}$ in lin-log plot $^{23}$ to estimate the relative contribution of the SWITG part of the $k_{y}$ spectrum to the overall thermal ion heat flux (note that $\int Q d k_{y}=\int Q k_{y} d \log k_{y}$ ). When the heat flux $Q\left(k_{y}\right)$ is multiplied by $k_{y}$ and plotted versus $\log k_{y}$, the area under the curve $k_{y} Q\left(k_{y}\right)$ becomes proportional to the corresponding contribution to the total flux, for a given interval of $\log k_{y}$, i.e. $\delta \log k_{y}$. One can indeed show that the area under the curve $k_{y} Q\left(k_{y}\right)$, for a given $\delta \log k_{y}$ is $\delta S_{Q k_{y}}=k_{y} Q\left(k_{y}\right) \delta \log k_{y}$ $=k_{y} Q\left(k_{y}\right) \delta k_{y} / k_{y}=Q\left(k_{y}\right) \delta k_{y}$, where $\delta \log k_{y}=\delta k_{y} / k_{y}$ Thus in addition to Fig. 3, $k_{y} Q\left(k_{y}\right)$ vs $k_{y}$ in lin-log plot has been depicted in Fig. 4. It is clear from the figure that the bulk of the thermal heat flux is contained in the lower $k_{y}$ part of the spectrum.

For completeness, we have also presented an estimate of transport based on a simple mixing length theory $\gamma / k_{\perp}^{2}$ using the linear growth rate $\gamma$ and perpendicular wavenumber $k_{y}$ in Fig. 5 by linear and log-log plots. However, similar to the results obtained in the nonlinear simulations the ratio of SWITG to ITG contributions to the heat transport is very small.

It is evident from Fig. 1 that the SWITG mode has growth rate comparable with the standard ITG mode for $R / L_{n}=10$ in the linear regime. On the other hand, for $R / L_{n}=5$ both humps become weaker, with the second hump corresponding to the SWITG mode being even weaker than the first hump corresponding to the standard ITG mode. Therefore, it is interesting to nonetheless estimate from the nonlinear simulation results the (small) contribution of the SWITG part of the $k_{y}$ spectrum to the net ion heat transport. To estimate the relative contribution of the SWITG mode to the ion heat flux compared to the standard ITG mode, we have calculated the cumulative sum of the time averaged heat flux $Q\left(k_{y}\right)$ given by

$$
Q_{c s}\left(k_{y 1}\right)=\sum_{k_{y}=k_{y m i n}}^{k_{y 1}} Q\left(k_{y}\right) .
$$

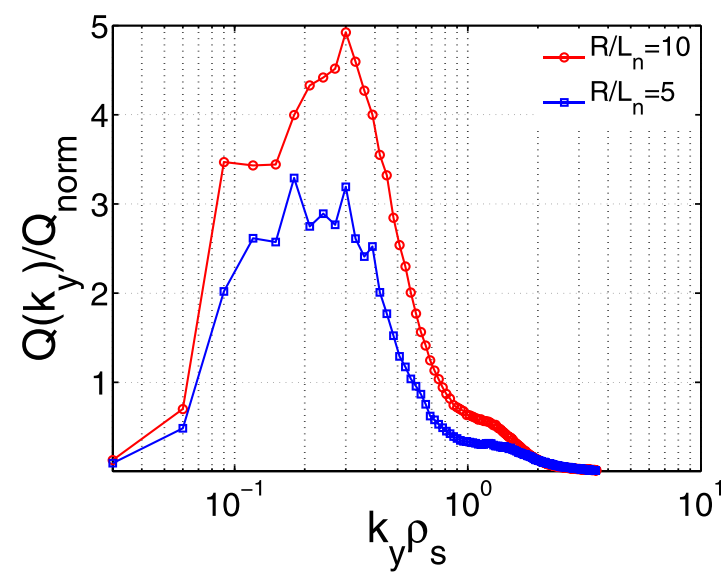

FIG. 4. Time averaged ion heat flux multiplied by normalized wavenumber $k_{y}$ versus normalized wavenumber $k_{y}$ for $R / L_{n}=5$ and $R / L_{n}=10$ in lin-log plot. $Q$ is normalized with respect to $Q_{\text {norm }}=p_{\text {ref }} c_{s} \rho_{s}^{2} / R^{2}$. 

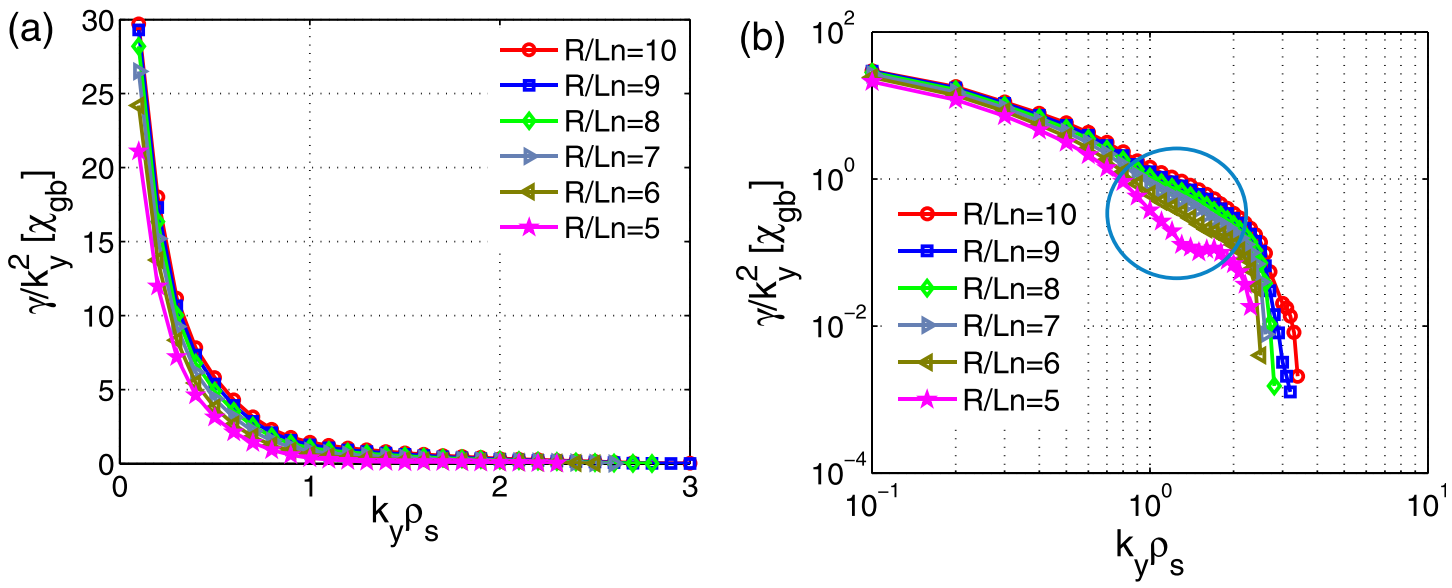

FIG. 5. Mixing length estimation of ion heat diffusivity versus normalized wavenumber $k_{y}$ in (a) lin-lin plot and (b) log-log plot, for linear runs displayed in Fig. 1. The circle shows the region where SWITG mode is unstable. Here, $\chi_{g b}=c_{s} \rho_{s}^{2} / R$.

It has been plotted versus $k_{y} \rho_{s}$ for the two cases $R / L_{n}=$ 10 and $R / L_{n}=5$ in Figs. 6 and 7 , respectively. It is apparent from the figures that $Q_{c s}$ increases rapidly for $k_{y} \rho_{s}<1$ and then tends to saturate for $k_{y} \rho_{s}>1$ in both cases. As expected, the cumulative heat flux in the case of $R / L_{n}=10$ is higher than for the case $R / L_{n}=5$. It is obvious from Figs. 2 and 3(a) that the heat flux in the case of $R / L_{n}=10$ is much higher than for $R / L_{n}=5$, leading to higher values of $Q_{c s}$. To evaluate the relative contribution to the net ion heat transport of the higher $k_{y}$ tail, $k_{y} \rho_{s} \geq 1$, corresponding to the SWITG mode, compared to the lower $k_{y}$ part, $k_{y} \rho_{s} \leq 1$, relevant to the standard ITG, one may compute

$$
\frac{Q_{c s}\left(k_{y} \rho_{s}\right)_{\max }-Q_{c s}\left(k_{y} \rho_{s}=1\right)}{Q_{c s}\left(k_{y} \rho_{s}=1\right)},
$$

where $\left(k_{y} \rho_{s}\right)_{\max }$ is the maximum wavenumber. One thus obtains that the net contribution of the SWITG part of the $k_{y}$ spectrum to the total ion heat flux is around 3.5\% in both cases in spite of the fact that linearly the SWITG mode for $R / L_{n}=10$ has growth rate more than double the one for $R / L_{n}=5$ and comparable to the ITG growth rate. Thus, even in the strongest cases of linear SWITG such as $R / L_{n}$ $=10$ considered here, the thermal ion heat flux is predominantly determined by the lower $k_{y}$ components of the fluctuation relevant to the standard ITG mode.

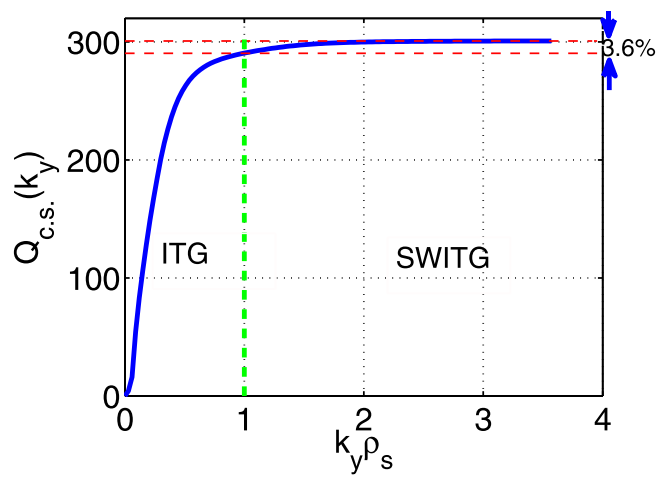

FIG. 6. Cumulative sum of heat flux for $R / L_{n}=10$. The standard ITG contribution to the heat flux is for $k_{y} \rho_{s} \leq 1$ and SWITG contribution to the heat flux is for $k_{y} \rho_{s} \geq 1$. $Q_{c s}$ is normalized with respect to $Q_{\text {norm }}=p_{r e f} c_{s} \rho_{s}^{2} / R^{2}$.
Snapshots of the electrostatic potential $\Phi_{1}(x, y)$ and $n_{1}(x, y)$ over the entire domain of $k_{y}$ spectrum measured at $t=330 R / c_{s}$ on the low field side $(z=0)$ of the tokamak during the nonlinear simulations with $R / L_{n}=5$ and $R / L_{n}=10$ are displayed in Figs. 8 and 9, respectively. It is clear from the figures that in the case of $R / L_{n}=5$, where SWITG is weak in the linear spectrum, the quantities $\Phi_{1}(x, y)$ and $n_{1}(x, y)$ exhibit elongated structures along the $x$ direction. The same quantities, in contrast, exhibit finer radial structures in the case of the nonlinear simulation with $R / L_{n}=10$, which has the highest growth rate in the linear phase. Given the elongated radial structures observed for $R / L_{n}=5$, which appear to be essentially broken up for $R / L_{n}=10$, one is motivated to investigate the zonal flow ${ }^{26-28}$ shearing rate in both cases. For that purpose, we have measured the zonal flow shearing rate in both simulations $R / L_{n}=5$ and $R / L_{n}=10$, respectively. The time evolution of the shearing rate is presented in Fig. 10 for the two nonlinear simulations. The estimated time average of the shearing rate $\omega_{E}=d v_{E y} / d x$ is $2.4 c_{s} / R$ for $R / L_{n}=5$ and $4.36 c_{s} / R$ for $R / L_{n}=10$, respectively. This means that the zonal flow shearing rate for the SWITG mode for $R / L_{n}=10$ is almost double the one for $R / L_{n}=5$. That is why the SWITG mode with $R / L_{n}=10$ exhibits finer structures, while that with $R / L_{n}=5$ bears more elongated structures in all perturbed quantities. In both cases, the shearing rate is much higher than the growth rate implying that zonal flows are the

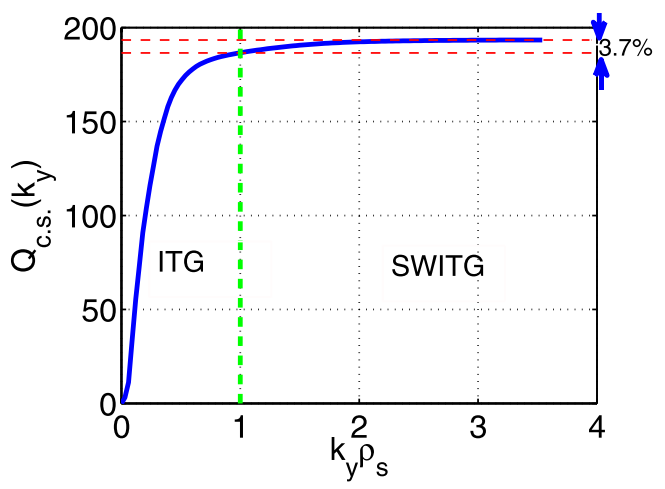

FIG. 7. Same as Fig. 6 but for $R / L_{n}=5$. 

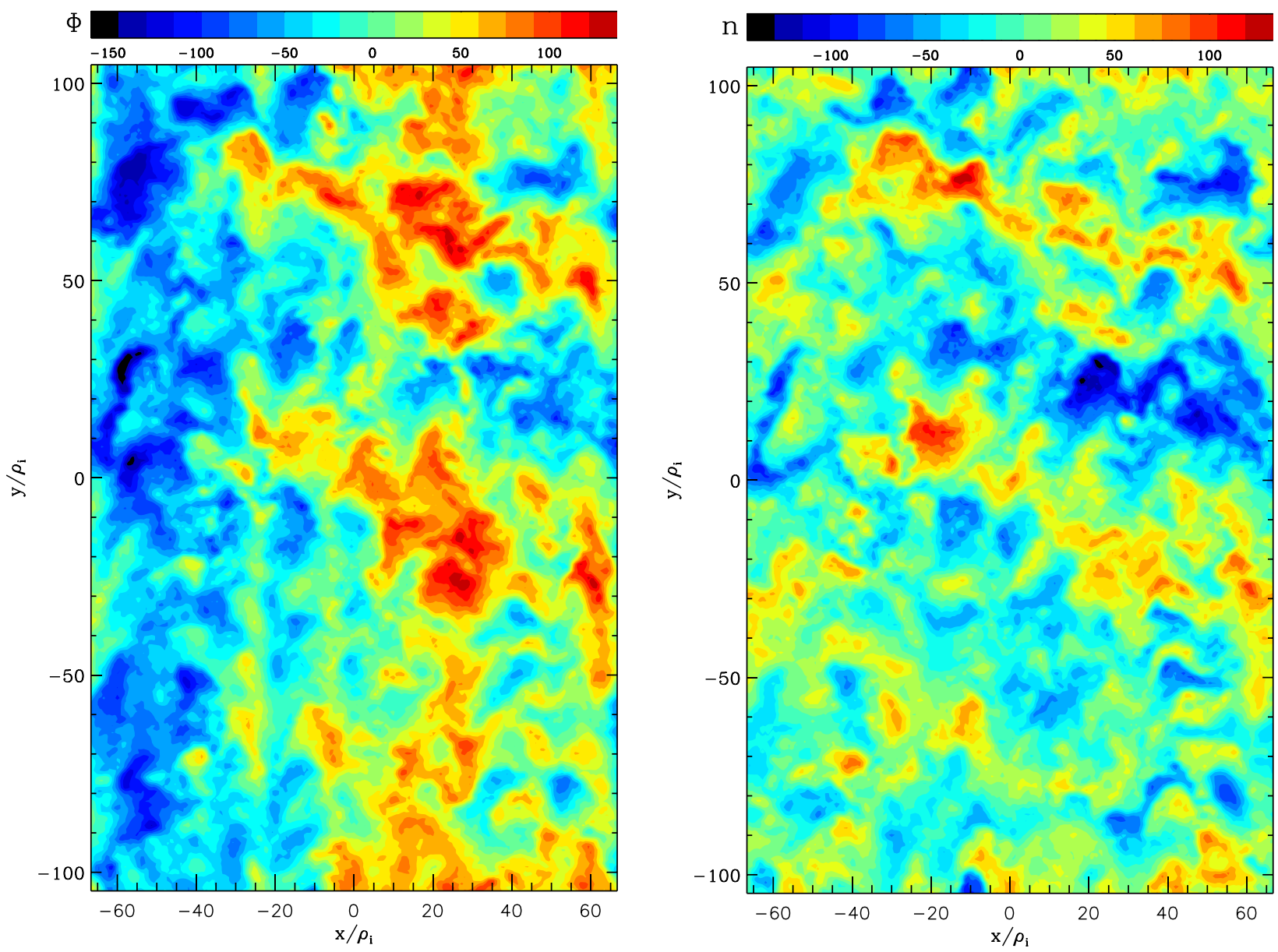

FIG. 8. Snapshots of the turbulent potential (left) and perturbed density (right) fields including the SWITG mode for $R / L_{n}=5$, taken at $t=330 R / c_{s}$.

dominant saturation mechanism for the SWITG mode turbulence. Note that the maximum linear growth rates of the SWITG mode are $0.32 c_{s} / R$ and $1.6 c_{s} / R$, respectively, for $R / L_{n}=5$ and $R / L_{n}=10$ at $k_{y} \rho_{s} \simeq 1.5$.

The saturation of the nonlinear SWITG mode can also be understood in terms of secondary instabilities. It may be noted that secondary instabilities are found to be responsible for nonlinear saturation of microinstabilities, ${ }^{3,21,29,30}$ which are then referred to as the primary instabilities. The secondary instabilities can be driven by the perpendicular shear in parallel velocity and/or perpendicular $E \times B$ velocities, and the level of saturation of a primary instability is determined by the dominant secondary instability. ${ }^{4}$ For example, curvature driven microinstabilities are saturated preferentially by the $E \times B$ shear driven secondary instability. ${ }^{4}$ However, the strength of this secondary instability depends on the nature of ions. When ions are adiabatic the $E \times B$ shear driven secondary instability becomes weaker, leading to elongated structures in the nonlinear phase of the primary instability. A much known example in this context is the ETG mode mo $^{3,4}$ where ions behave adiabatically. Since in the present case, even at $k_{\perp}^{2} \rho_{s}^{2}>1$ the ions are nonadiabatic because of steeper density profiles, the $E \times B$ shear driven secondary instabilities may be the major saturation mechanism that regulates the primary SWITG instability.

From the present linear and nonlinear simulations, one arrives at two conclusions: (1) Although linearly the growth rate of the SWITG mode is much higher for $R / L_{n}=10$ than for $R / L_{n}=5$, the nonlinear contribution to the overall ion heat flux is same in both cases $(\simeq 3.5 \%)$; the nonlinear SWITG mode for $R / L_{n}=10$ exhibits higher rate of zonal flow and hence finer structure than the one for $R / L_{n}=5$. (2) Although linearly SWITG is stronger than the ITG mode for $R / L_{n} \geq 10$, the bulk part of ion heat fluxes comes from the ITG mode. Both these results can be explained in terms of the role of secondary instabilities.

Let us first discuss the first point. Since at $k_{\perp}^{2} \rho_{s}^{2}>1$ the ions are more adiabatic for $R / L_{n}=5.0$ than those for $R / L_{n}=10.0$ as $\omega_{* i}$ is smaller in the former case, the $E \times B$ shear driven secondary instability also is therefore weaker for $R / L_{n}=5.0$. Therefore, one observes higher shearing rate and finer structures for $R / L_{n}=10.0$ than for $R / L_{n}=5.0$. Hence the contribution of the SWITG mode for $R / L_{n}=10.0$ to the overall ion heat flux is almost as low as the one for $R / L_{n}=5$, even though the latter has the weakest growth rate among all values of $R / L_{n}$ considered in the linear simulation.

Next, we come to the second point. The $E \times B$ shear driven instability scales as $k_{y}^{2} \Phi_{L},{ }^{4}$ where $\Phi_{L}$ is the amplitude of the linear SWITG mode. Note that the values of $k_{y}$ 's where the linear growth rate of the ITG and SWITG modes peak is roughly 0.6 and 1.5 , respectively (Fig. 1), and the corresponding growth rates have comparable magnitude. Therefore, SWITG modes are sub-dominant nonlinearly as their higher growth rates are overcompensated by even larger 

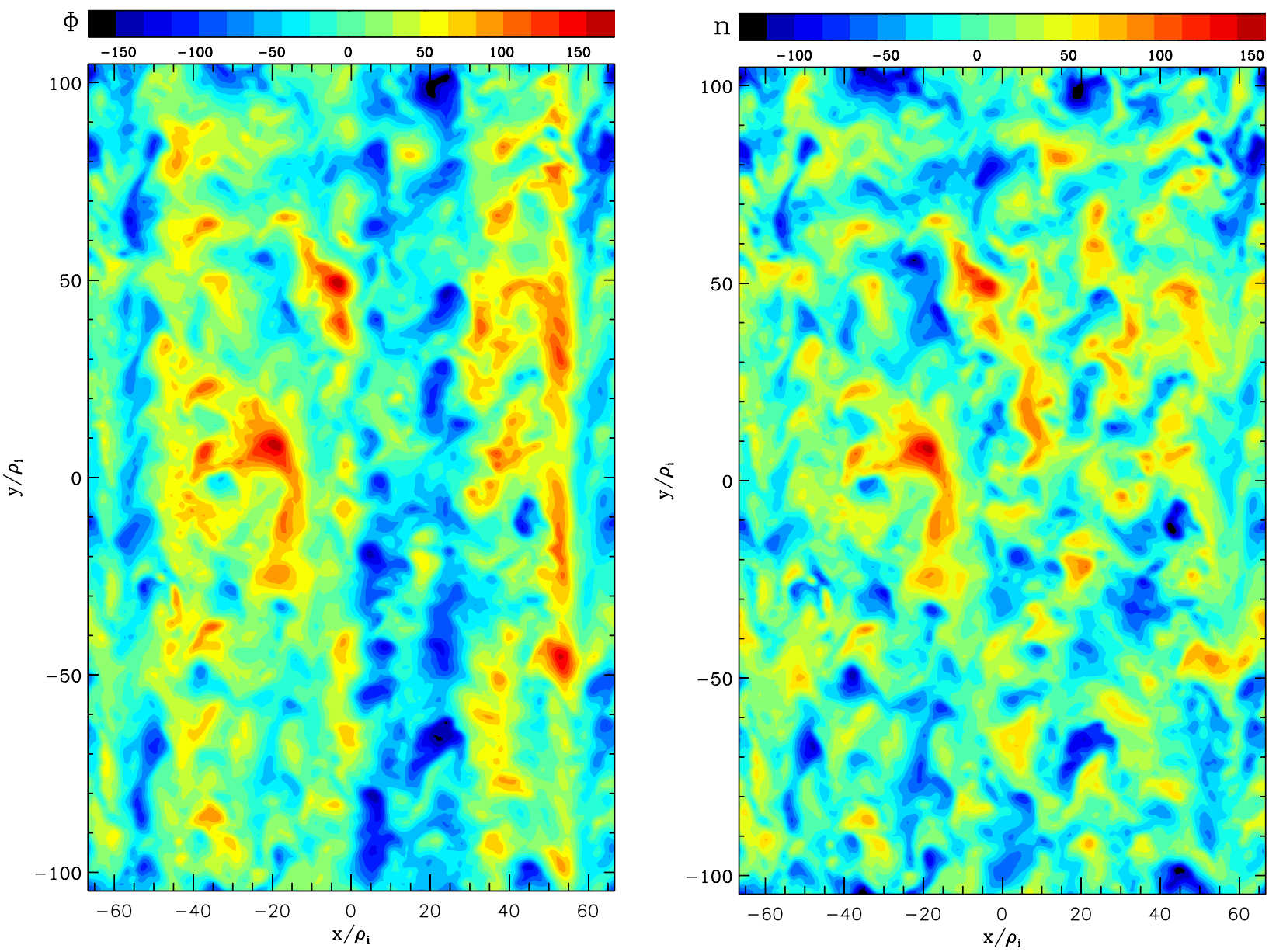

FIG. 9. Snapshots of the turbulent potential (left) and perturbed density (right) fields including the SWITG mode for $R / L_{n}=10$, taken at $t=330 R / c_{s}$.
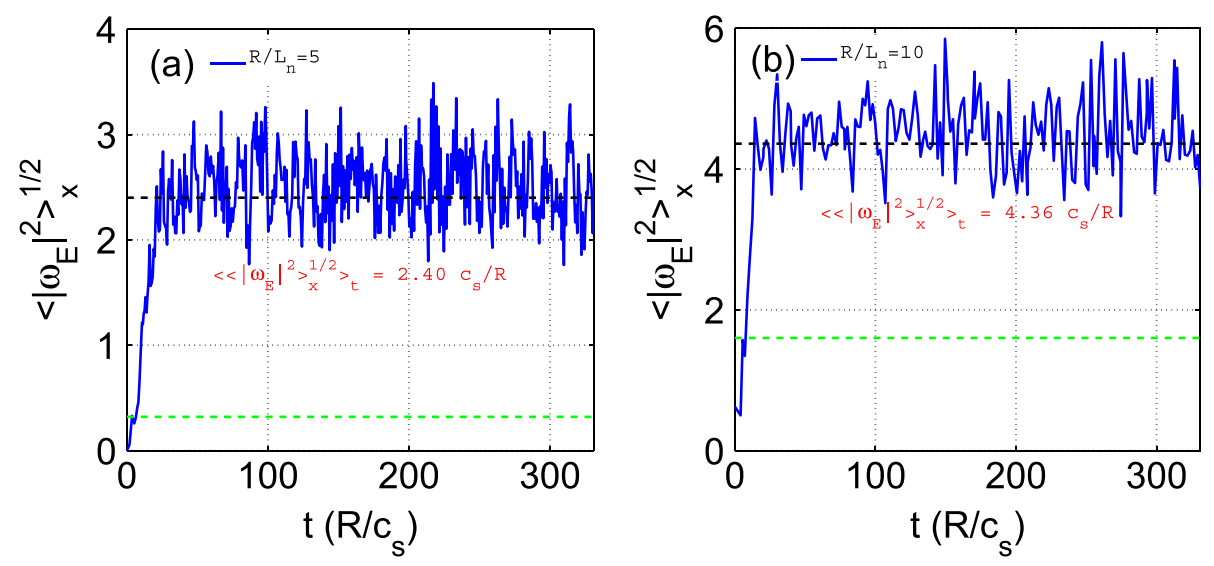

FIG. 10. Time traces of the zonal flow shearing rate $\omega_{E}=d v_{E, y} / d x$, in units of $c_{s} / R$ for the nonlinear simulations with (a) $R / L_{n}=5$ and (b) $R / L_{n}=10$. The green lines represent the corresponding maximum linear growth rates for the SWITG mode, i.e., $0.32 c_{s} / R$ and $1.6 c_{s} / R$, respectively, for $R / L_{n}=5$ and $R / L_{n}=10$. nonlinear growth rates (proportional to $k_{y}^{2}$ ) of secondary instabilities. In the standard ITG part of the $k_{y}$ spectrum, the strength of the secondary instability is smaller compared to SWITG part, as $k_{y}$ is smaller in the former case. The suppression level of ITG in the nonlinear regime is therefore much weaker compared to SWITG, thereby contributing much higher to the overall thermal ion heat flux.

\section{CONCLUSION}

In the present work, the short wavelength ion temperature gradient mode has been studied linearly and nonlinearly using the flux tube version of the massively parallel, gyrokinetic code GENE. Linearly the mode appears to be as dominant as the standard ITG mode for high $R / L_{n} \sim 10$. However, the growth rate decreases with decreasing $R / L_{n}$, and below $R / L_{n}=5$ the SWITG mode vanishes, i.e., only the standard ITG mode remains unstable. Nonlinear simulations show higher heat fluxes for higher values of $R / L_{n}$, consistent with the trend in the linear growth rate with respect to $R / L_{n}$. The nonlinear heat flux spectrum is peaked at lower $k_{y} \rho_{s} \simeq 0.1$. The estimate of relative contribution of the higher $k_{y} \rho_{s}$ part of the heat flux spectrum corresponding to the SWITG mode reveals that the contribution of the SWITG 
mode to the total heat flux is very low (around 3.5\%) compared to the contribution from the lower $k_{y}$ part of the spectrum typically belonging to the standard ITG mode. The shearing rate has been found to be much higher than the linear mode frequency and growth rate of the SWITG mode, suggesting that the zonal flows are the main saturation mechanisms of the SWITG turbulence. This is in conformity with the results of Gao et al. ${ }^{18}$ which reported that the slab SWITG mode can be stabilized by $E \times B$ velocity shear. Also, in our case, the shearing rate is found to be higher for the SWITG modes with higher $R / L_{n}$ which also exhibit higher growth rate linearly compared to the shearing rate for those with lower $R / L_{n}$ and lower growth rate linearly. The higher shearing rate appears to compensate for the higher growth rate of the mode, leading to a minimal contribution to the thermal ion heat flux from the higher $k_{y} \rho_{s}$ part of the spectrum relevant to the SWITG mode, irrespective of the density scale length which determines the strength of the SWITG mode with respect to the standard ITG linearly.

Although SWITG has been found to have very small contribution to net thermal ion heat flux, it can be important for electron thermal transport when coupled to other modes, such as trapped electrons modes (TEM) that is in the presence of nonadiabatic electrons at higher $k_{y}$ scales. Thus, the smallness of the contribution of SWITG modes to heat transport obtained here with an adiabatic electron model, might be different when considering nonadiabatic or kinetic electron dynamics and interaction with trapped electron modes. This issue will be addressed in a future communication.

\section{ACKNOWLEDGMENTS}

Author J.C. is thankful to the Indo-Swiss Joint Research Programme (2008-2009) in the frame of which this work was carried out. Entire computational work was performed on the Pleiades2 Linux cluster of CRPP-EPFL.
${ }^{1}$ W. Horton, Rev. Mod. Phys. 71, 735 (1999).

${ }^{2}$ P. N. Guzdar, C. S. Liu, J. Q. Dong, and Y. C. Lee, Phys. Rev. Lett. 57, 2818 (1986).

${ }^{3}$ W. Dorland, F. Jenko, M. Kotchenreuther, and B. N. Roger, Phys. Rev. Lett. 85, 5579 (2000).

${ }^{4}$ F. Jenko and W. Dorland, Phys. Rev. Lett. 89, 225001 (2002).

${ }^{5}$ C. Z. Cheng and L. Chen, Phys. Fluids 23, 1770 (1980).

${ }^{6}$ J. Chowdhury, R. Ganesh, S. Brunner, J. Vaclavik, and L. Villard, Phys. Plasmas 17, 102105 (2010).

${ }^{7}$ B. Coppi and G. Rewoldt, Phys. Rev. Lett. 33, 1329 (1974).

${ }^{8}$ T. Dannert and F. Jenko, Phys. Plasmas 12, 072309 (2005).

${ }^{9}$ W. X. Wang, T. S. Hahm, S. Ethier, L. E. Zakharov, and P. H. Diamond, Phys. Rev. Lett. 106, 085001 (2011).

${ }^{10}$ F. Romanelli, Phys. Fluids B 1, 1018 (1989).

${ }^{11}$ J. Chowdhury, R. Ganesh, P. Angelino, J. Vaclavik, L. Villard, and S. Brunner, Phys. Plasmas 15, 072117 (2008).

${ }^{12}$ A. I. Smolyakov, M. Yagi, and Y. Kishimoto, Phys. Rev. Lett. 89, 125005-1 (2002).

${ }^{13}$ A. Hirose, M. Elia, A. I. Smolyakov, and M. Yagi, Phys. Plasmas 9, 1659 (2002).

${ }^{14}$ Z. Gao, H. Sanuki, K. Itoh, and J. Q. Dong, Phys. Plasmas 10, 2831 (2003).

${ }^{15}$ Z. Gao, H. Sanuki, K. Itoh, and J. Q. Dong, Phys. Plasmas 12, 022502-1 (2005).

${ }^{16}$ J. Chowdhury, R. Ganesh, J. Vaclavik, S. Brunner, L. Villard, and P. Angelino, Phys. Plasmas 16, 082511 (2009).

${ }^{17}$ S. Brunner, M. Fivaz, T. M. Tran, and J. Vaclavik, Phys. Plasmas 5(11), 3929 (1998).

${ }^{18}$ Z. Gao, J. Q. Dong, and H. Sanuki, Phys. Plasmas 11, 3053 (2004).

${ }^{19}$ Z. Gao, H. Sanuki, K. Itoh, and J. Q Dong, J. Plasma Phys. 72, 1249 (2006).

${ }^{20}$ Yi.-K. Pu and S. Migliuolo, Phys. Fluids 28, 1722 (1985).

${ }^{21}$ F. Jenko, W. Dorland, M. Kotschenreuther, and B. N. Rogers, Phys. Plasmas 7, 1904 (2000).

${ }^{22}$ T. Görler, X. Lapillonne, S. Brunner, T. Dannert, F. Jenko, F. Merz, and D. Told, J. Comput. Phys. 230, 7053 (2011).

${ }^{23}$ T. Görler and F. Jenko, Phys. Rev. Lett. 100, 185002 (2008).

${ }^{24}$ X. Lapillonne, S. Brunner, T. Dannert, S. Jolliet, A. Marinoni, L. Villard, T. Görler, F. Jenko, and F. Merz. Phys. Plasmas 16, 032308 (2009).

${ }^{25}$ R. C. Wolf, Plasma Phys. Controlled Fusion 45, R1-R91 (2003).

${ }^{26}$ Z. Lin, T. S. Hahm, W. W. Lee, W. M. Tang, and R. B. White, Science 281, 1835 (1998).

${ }^{27}$ B. N. Rogers, W. Dorland, and M. Kotschenreuther, Phys. Rev. Lett. 85, 5336 (2000).

${ }^{28}$ P. H. Diamond, S. Itoh, K. Itoh, and T. S. Hahm, Plasma Phys. Controlled Fusion 47, R35 (2005).

${ }^{29}$ D. Strintzi and F. Jenko, Phys. Plasmas 14, 042305 (2007).

${ }^{30}$ F. Jenko, Phys. Lett. A 351, 417 (2006). 